\title{
Retraction Note to: Risk factors for increased left ventricular hypertrophy in patients with chronic kidney disease
}

\author{
Kosaku Nitta ${ }^{1} \cdot$ Satoshi Iimuro $^{2} \cdot$ Enyu Imai $^{3} \cdot$ Seiichi Matsuo $^{3} \cdot$ Hirofumi Makino $^{4}$. \\ Tadao Akizawa $^{5}$. Tsuyoshi Watanabe ${ }^{6}$ - Yasuo Ohashi ${ }^{2} \cdot$ Akira Hishida $^{7}$
}

Published online: 9 September 2017

(C) Japanese Society of Nephrology 2017

\section{Retraction to: Clin Exp Nephrol (2013) 17:730-742 \\ DOI 10.1007/s10157-012-0758-4}

The editorial board has decided to retract the above-cited article entitled "Risk factors for increased left ventricular hypertrophy in patients with chronic kidney disease", doi:10.1007/s10157-012-0758-4, because of an inadvertent but serious error.

Upon carrying out further analyses, the authors discovered that the program used in merging their data set was not working correctly, which may have made the conclusion stated in their article questionable.

The online version of the original article can be found under doi:10.1007/s10157-012-0758-4.

Kosaku Nitta

knitta@kc.twmu.ac.jp

1 Department of Medicine, Kidney Center, Tokyo Women's Medical University, 8-1 Kawada-cho, Shinjuku-ku,

Tokyo 162-8666, Japan

2 Department of Biostatistics, School of Public Health, The University of Tokyo, Tokyo, Japan

3 Department of Nephrology, Nagoya University Graduate School of Medicine, Nagoya, Japan

4 Department of Medicine and Clinical Science, Okayama University Graduate School of Medicine, Dentistry and Pharmaceutical Sciences, Okayama, Japan

5 Department of Nephrology, Showa University, Tokyo, Japan

6 Third Department of Internal Medicine, Fukushima Medical University, Fukushima, Japan

7 Yaizu City Hospital, Shizuoka, Japan 